Research Article

\title{
On the normalized (distance) Laplacian spectrum of linear dependence graph of a finite- dimensional vector space
}

\author{
Jinu Mary Jameson ${ }^{1,2}$, Gopalapilla Indulal ${ }^{3, *}$ \\ ${ }^{1}$ Department of Mathematics, St. Berchmans College, Changanassery, Kottayam, India \\ ${ }^{2}$ Department of Mathematics, Marthoma College, Thiruvalla, Pathanamthitta, India \\ ${ }^{3}$ Department of Mathematics, St. Aloysius College, Edathua, Alappuzha, India
}

(Received: 11 November 2020. Received in revised form: 17 February 2021. Accepted: 3 March 2021. Published online: 9 March 2021.)

(C) 2021 the authors. This is an open access article under the CC BY (International 4.0) license (www.creativecommons.org/licenses/by/4.0/)

\begin{abstract}
Let $W$ be a finite-dimensional vector space over a finite field $F$. The linear dependence graph $\Gamma(W)$ of $W$ is a simple graph whose vertices are the elements of the vector space $W$ and two distinct vertices $u, v$ of the graph $\Gamma(W)$ are adjacent if and only if $u$ and $v$ are linearly dependent in $W$. In this paper, we describe the normalized Laplacian spectrum and normalized distance Laplacian spectrum of the graph $\Gamma(W)$.
\end{abstract}

Keywords: linear dependence graph; normalized Laplacian matrix ; normalized Laplacian spectrum; normalized distance Laplacian matrix; normalized distance Laplacian spectrum.

2020 Mathematics Subject Classification: 05C12, 05C50.

\section{Introduction}

The existing literature on algebraic graph theory gives different kinds of spectra associated with a graph. Spectral analysis of graphs has been the subject of considerable research in algebraic graph theory from the pioneering work of Hückel [5]. The study of different algebraic structures, using the properties of graphs associated to them, is also a well-studied topic. In this paper, we associate a finite-dimensional vector space over a finite field to a simple graph by using the linear dependency of two vectors in the considered space.

Let $F$ be a finite field with $q$ elements and $W$ be a finite-dimensional vector space over $F$ with dimension $n$. Hence, the vector space $W$ consists of $q^{n}$ elements. Two finite-dimensional vector spaces over $F$ are isomorphic if and only if they have the same dimension. The linear dependence graph $\Gamma(W)$ associated to the vector space $W(F)$ is defined [6] as the graph with vertex set $W$ where two vertices $u, v$ of the graph $\Gamma(W)$ are adjacent if and only if $u$ and $v$ are linearly dependent in $W$. The linear dependence graph of the 2-dimensional vector space $W=\{(0,0),(0,1),(1,0),(2,0),(0,2),(1,1),(2,2),(1,2),(2,1)\}$ over the finite field $F_{3}=\{0,1,2\}$ is shown in Figure 1.

The adjacency spectrum of $\Gamma(W)$ was found in [6]. The study of graphs related to algebraic structures along the spectral aspect gained momentum through the work of Maity and Bhuniya [6]. For some recent works along these lines, see [4, 8,9]. In this paper, we obtain the normalized Laplacian and normalized distance Laplacian spectra of $\Gamma(W)$.

Let $G=(V, E)$ be a simple graph with vertex set $V(G)=\left\{v_{1}, v_{2}, \ldots, v_{n}\right\}$. Denote by $D(G)$ the diagonal matrix of $G$ (also known as the degree matrix of $G$ ). The adjacency matrix of $G$ is the matrix $A(G)=\left[a_{i j}\right], 1 \leq i, j \leq n$, where $a_{i j}=1$ if $v_{i}$ adjacent to $v_{j}$ and $a_{i j}=0$ otherwise. The Laplacian matrix $L(G)$ is defined as $L(G)=D(G)-A(G)$. The normalized Laplacian matrix $\mathcal{L}(G)$ was introduced in [3] as the square matrix whose $(i, j)$-entry $\mathcal{L}_{i j}, 1 \leq i, j \leq n$, is given as

$$
\mathcal{L}_{i j}= \begin{cases}1 & \text { if } v_{i}=v_{j} \text { and } d_{v_{i}} \neq 0, \\ \frac{-1}{\sqrt{d_{v_{i}} d_{v_{j}}}} & \text { if } v_{i} \text { and } v_{j} \text { are adjacent } \\ 0 & \text { otherwise, }\end{cases}
$$

where $d_{v_{i}}$ and $d_{v_{j}}$ are degrees of $v_{i}$ and $v_{j}$, respectively. We can also write

$$
\mathcal{L}(G)=D^{-1 / 2}(G) L(G) D^{-1 / 2}(G)=I-D^{-1 / 2}(G) A(G) D^{-1 / 2}(G)
$$

*Corresponding author (indulalgopal@aloysiuscollege.ac.in). 


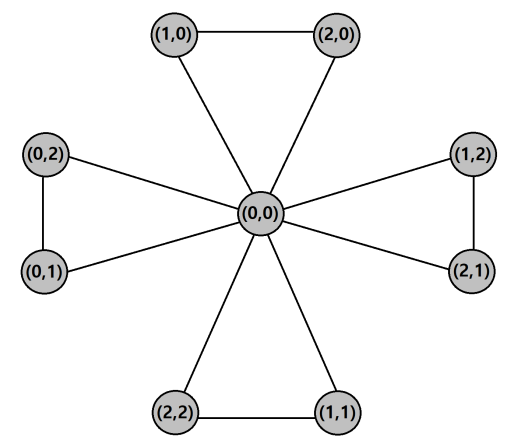

Figure 1: The linear dependence graph of the 2-dimensional vector space $W$ over the finite field $F_{3}=\{0,1,2\}$ where $W=\{(0,0),(0,1),(1,0),(2,0),(0,2),(1,1),(2,2),(1,2),(2,1)\}$.

where $A(G), L(G)$ and $D(G)$ are the adjacency matrix, Laplacian matrix and degree matrix respectively of the graph $G$ with the convention that $D(G)^{-1}(u, u)=0$ if $d_{u}=0$. The eigenvalues of $\mathcal{L}(G)$ are called normalized Laplacian eigenvalues of $G$ and forms the $\mathcal{L}$-spectrum of $G$. Two graphs are said to be $\mathcal{L}$-cospectral if they have the same $\mathcal{L}$-spectrum. In [3], Chung and Graham showed that $\mathcal{L}(G)$ is a positive definite symmetric matrix and all its eigenvalues lie within the interval $[0,2]$ and that 0 is always a normalized Laplacian eigenvalue of any graph $G$.

Let $P(G)=D(G)^{-1} A(G)$. Then, $\mathcal{L}(G)=D(G)^{1 / 2}\left(I-D(G)^{-1} A(G)\right) D(G)^{-1 / 2}=D(G)^{1 / 2}(I-P(G)) D(G)^{-1 / 2}$. Hence, if the spectrum of $P(G)$ is $\left\{\mu_{1}, \mu_{2}, \ldots, \mu_{n}\right\}$, then the spectrum of $\mathcal{L}(G)$ is $\left\{\left(1-\mu_{1}\right),\left(1-\mu_{2}\right), \ldots,\left(1-\mu_{n}\right)\right\}$.

The distance matrix $\mathcal{D}(G)$ is the matrix whose $(i, j)$-entry is equal to $d_{G}\left(v_{i}, v_{j}\right)$, the distance between the vertices $v_{i}$ and $v_{j}$ of $G$. The eigenvalues of $\mathcal{D}(G)$ are said to be the $\mathcal{D}$-eigenvalues of $G$. The transmission $\operatorname{Tr}(v)$ of a vertex $v$ of $G$ is the sum of the distances from $v$ to all other vertices of $G$ and the transmission matrix $T(G)$ is the diagonal matrix defined as $T(G)=\operatorname{diag}\left(\operatorname{Tr}\left(v_{1}\right), \operatorname{Tr}\left(v_{2}\right), \ldots, \operatorname{Tr}\left(v_{n}\right)\right)$. The distance Laplacian matrix is defined as $\mathcal{D}^{L}(G)=T(G)-\mathcal{D}(G)$. The normalized distance Laplacian matrix is defined [7] as $\mathcal{D}^{N}(G)=T(G)^{-1 / 2} \mathcal{D}^{L}(G) T(G)^{-1 / 2}$. Eigenvalues of $\mathcal{D}^{N}(G)$ are called normalized distance Laplacian eigenvalues of $G$ and forms the $\mathcal{D}^{N}$-spectrum of $G$. Two graphs are said to be $\mathcal{D}^{N}$-cospectral if they have same $\mathcal{D}^{N}$-spectrum. A graph $G$ is said to be a $r$-transmission regular graph if $\operatorname{Tr}(v)=r$ for every vertex $v$ of $G$. If $G$ is $r$-transmission regular then,

$$
\mathcal{D}^{L}(G)=r I-\mathcal{D}(G) \quad \text { and } \quad \mathcal{D}^{N}(G)=T(G)^{-1 / 2}(T(G)-\mathcal{D}(G)) T(G)^{-1 / 2}=I-\frac{\mathcal{D}(G)}{r} .
$$

Let $\bar{P}(G)=T(G)^{-1} \mathcal{D}(G)$. Then $\mathcal{D}^{N}(G)=T(G)^{1 / 2}\left(I-T(G)^{-1} \mathcal{D}(G)\right) T(G)^{-1 / 2}=T(G)^{1 / 2}(I-\bar{P}(G)) T(G)^{-1 / 2}$. Hence if the spectrum of $\bar{P}(G)$ is $\left\{\mu_{1}^{\prime}, \mu_{2}^{\prime}, \ldots, \mu_{n}^{\prime}\right\}$, then the spectrum of $\mathcal{D}^{N}(G)$ is $\left\{\left(1-\mu_{1}^{\prime}\right),\left(1-\mu_{2}^{\prime}\right), \ldots,\left(1-\mu_{n}^{\prime}\right)\right\}$.

Since we are dealing with the graph $\Gamma(W)$ throughout this paper $A(\Gamma(W)), D(\Gamma(W)), P(\Gamma(W)), \mathcal{D}(\Gamma(W)), T(\Gamma(W))$, $\bar{P}(\Gamma(W))$ are denoted as simply $A, D, P, \mathcal{D}, T, \bar{P}$, respectively. Also, $I_{n}$ denotes the identity matrix of order $n, O_{n}$ denotes the zero matrix of order $n, J_{n}$ denotes all-one matrix of order $n$ and $J_{m \times n}$ denotes the all-one matrix of order $m \times n$. All spectral graph theoretic terminology are taken from [3]. We use the following lemma in the next section.

Lemma 1.1. [2] Let $M, N, P$ and $Q$ be matrices such that $M$ invertible. Let

$$
S=\left[\begin{array}{ll}
M & N \\
P & Q
\end{array}\right]
$$

Then $|S|=|M|\left|Q-P M^{-1} N\right|$ and if $M$ and $P$ commutes, then $|S|=|M Q-P N|$ where the symbol $|$.$| denotes the determi-$ nant.

\section{The normalized Laplacian spectrum of the linear dependence graph $\Gamma(W)$}

In this section, we find the normalized Laplacian spectrum of $\Gamma(W)$ in terms of $q$ by using the spectrum of $P$.

Theorem 2.1. Let $F$ be a finite field of order $q \geq 2$ and $W$ be an n-dimensional vector space over $F$. Then, the spectrum of $P$ is

$$
\left(\begin{array}{ccc}
1 & -(q-1)^{-1} & (q-2)(q-1)^{-1} \\
1 & k(q-2)+1 & k-1
\end{array}\right),
$$

where $k=q^{n-1}+q^{n-2}+\ldots+q+1$. 
Proof. In [6], the adjacency matrix of $\Gamma(W)$ was given as

$$
\left.A=\left[\begin{array}{cccccccccccccc}
0 & 1 & 1 & \cdots & 1 & 1 & 1 & \cdots & 1 & \cdots & 1 & 1 & \cdots & 1 \\
1 & 0 & 1 & \cdots & 1 & 0 & 0 & \cdots & 0 & \cdots & 0 & 0 & \cdots & 0 \\
1 & 1 & 0 & \cdots & 1 & 0 & 0 & \cdots & 0 & \cdots & 0 & 0 & \cdots & 0 \\
\vdots & \vdots & \vdots & \ddots & \vdots & \vdots & \vdots & \ddots & \vdots & \ddots & \vdots & \vdots & \ddots & \vdots \\
1 & 1 & 1 & \cdots & 0 & 0 & 0 & \cdots & 0 & \cdots & 0 & 0 & \cdots & 0 \\
1 & 0 & 0 & \cdots & 0 & 0 & 1 & \cdots & 1 & \cdots & 0 & 0 & \cdots & 0 \\
1 & 0 & 0 & \cdots & 0 & 1 & 0 & \cdots & 1 & \cdots & 0 & 0 & \cdots & 0 \\
\vdots & \vdots & \vdots & \ddots & \vdots & \vdots & \vdots & \ddots & \vdots & \ddots & \vdots & \vdots & \ddots & \vdots \\
1 & 0 & 0 & \cdots & 0 & 1 & 1 & \cdots & 0 & \cdots & 0 & 0 & \cdots & 0 \\
\vdots & \vdots & \vdots & \vdots & \vdots & \vdots & \vdots & \vdots & \vdots & \ddots & \vdots & \vdots & \vdots & \vdots \\
1 & 0 & 0 & \cdots & 0 & 0 & 0 & \cdots & 0 & \cdots & 0 & 1 & \cdots & 1 \\
1 & 0 & 0 & \cdots & 0 & 0 & 0 & \cdots & 0 & \cdots & 1 & 0 & \cdots & 1 \\
\vdots & \vdots & \vdots & \ddots & \vdots & \vdots & \vdots & \ddots & \vdots & \ddots & \vdots & \vdots & \ddots & \vdots \\
1 & 0 & 0 & \cdots & 0 & 0 & 0 & \cdots & 0 & \cdots & 1 & 1 & \cdots & 0
\end{array}\right]=\left[\begin{array}{ccc}
0 & \\
0 & \\
J_{1 \times\left(q^{n}-1\right)}
\end{array}\right] \otimes I_{q-1} I_{k}\right]
$$

where $k=q^{n-1}+q^{n-2}+\ldots+q+1$. The degree of the zero vector is $q^{n}-1$ and degree of all other vectors in $W$ is $q-1$. Hence the degree matrix of $\Gamma(W)$ is given by

$$
D=\left[\begin{array}{ccccc}
q^{n}-1 & 0 & 0 & \cdots & 0 \\
0 & q-1 & 0 & \cdots & 0 \\
0 & 0 & q-1 & \cdots & 0 \\
\vdots & \vdots & \vdots & \ddots & \vdots \\
0 & 0 & 0 & \cdots & q-1
\end{array}\right]=\left[\begin{array}{cc}
q^{n}-1 & 0_{1 \times\left(q^{n}-1\right)} \\
0_{\left(q^{n}-1\right) \times 1} & (q-1) I_{q-1} \otimes I_{k}
\end{array}\right]
$$

where $k=q^{n-1}+q^{n-2}+\ldots+q+1$. Hence,

$$
\begin{aligned}
P=D^{-1} A & =\left[\begin{array}{cc}
\left(q^{n}-1\right)^{-1} & 0_{1 \times\left(q^{n}-1\right)} \\
0_{\left(q^{n}-1\right) \times 1} & (q-1)^{-1} I_{q-1} \otimes I_{k}
\end{array}\right]\left[\begin{array}{cc}
0 & J_{1 \times\left(q^{n}-1\right)} \\
J_{\left(q^{n}-1\right) \times 1} & {\left[J_{q-1}-I_{q-1}\right] \otimes I_{k}}
\end{array}\right] \\
& =\left[\begin{array}{cc}
\left(q^{n}-1\right)^{-1} J_{1 \times\left(q^{n}-1\right)} \\
\left.(q-1)^{-1} I_{q-1} \otimes I_{k}\right) J_{\left(q^{n}-1\right) \times 1} & {\left[(q-1)^{-1} I_{q-1} \otimes I_{k}\right]\left(\left[J_{q-1}-I_{q-1}\right] \otimes I_{k}\right)}
\end{array}\right] \\
& =\left[\begin{array}{cc}
0 & \left(q^{n}-1\right)^{-1} J_{1 \times\left(q^{n}-1\right)} \\
(q-1)^{-1} J_{\left(q^{n}-1\right) \times 1} & (q-1)^{-1}\left[J_{q-1}-I_{q-1}\right] \otimes I_{k}
\end{array}\right] .
\end{aligned}
$$

The characteristic polynomial of $P$ is

$$
\begin{aligned}
\left(\lambda I_{q^{n}}-P\right) & =\left|\begin{array}{cc}
\lambda & -\left(q^{n}-1\right)^{-1} J_{1 \times\left(q^{n}-1\right)} \\
-(q-1)^{-1} J_{\left(q^{n}-1\right) \times 1} & \lambda I_{q^{n}-1}-\left((q-1)^{-1}\left[J_{q-1}-I_{q-1}\right] \otimes I_{k}\right)
\end{array}\right| \\
& =\left(q^{n}-1\right)^{-1}(q-1)^{-\left(q^{n}-1\right)}\left|\begin{array}{cc}
\lambda\left(q^{n}-1\right) & -J_{1 \times\left(q^{n}-1\right)} \\
-J_{\left(q^{n}-1\right) \times 1} & \left([\lambda(q-1)+1] I_{q-1}-J_{q-1}\right) \otimes I_{k}
\end{array}\right| .
\end{aligned}
$$

Multiplying the first row by $\lambda(q-1)-(q-2)$ and $R_{1}^{\prime} \mapsto R_{1}^{\prime}+R_{2}+\ldots+R_{q^{n}}$ and applying Lemma 1.1 yield

$$
\begin{aligned}
\operatorname{det}\left(\lambda I_{q^{n}}-P\right) & =\frac{\left(q^{n}-1\right)^{-1}(q-1)^{-\left(q^{n}-1\right)}}{\lambda(q-1)-(q-2)}\left|\begin{array}{cc}
\lambda\left(q^{n}-1\right)[\lambda(q-1)-(q-2)]-\left(q^{n}-1\right) & 0_{1 \times\left(q^{n}-1\right)} \\
-J_{\left(q^{n}-1\right) \times 1} & \left.(\lambda(q-1)+1] I_{q-1}-J_{q-1}\right) \otimes I_{k}
\end{array}\right| \\
& =\frac{\left(q^{n}-1\right)^{-1}(q-1)^{-\left(q^{n}-1\right)}}{\lambda(q-1)-(q-2)}\left[\lambda\left(q^{n}-1\right)[\lambda(q-1)-(q-2)]-\left(q^{n}-1\right)\right]\left[\operatorname{det}\left([\lambda(q-1)+1] I_{q-1}-J_{q-1}\right)\right]^{k} .
\end{aligned}
$$

If $A_{1}=\operatorname{det}\left([\lambda(q-1)+1] I_{q-1}-J_{q-1}\right)$ then

$$
A_{1}=\left|\begin{array}{cccc}
\lambda(q-1) & -1 & \cdots & -1 \\
-1 & \lambda(q-1) & \cdots & -1 \\
\vdots & \vdots & \ddots & \vdots \\
-1 & -1 & \cdots & \lambda(q-1)
\end{array}\right|_{(q-1) \times(q-1)}
$$




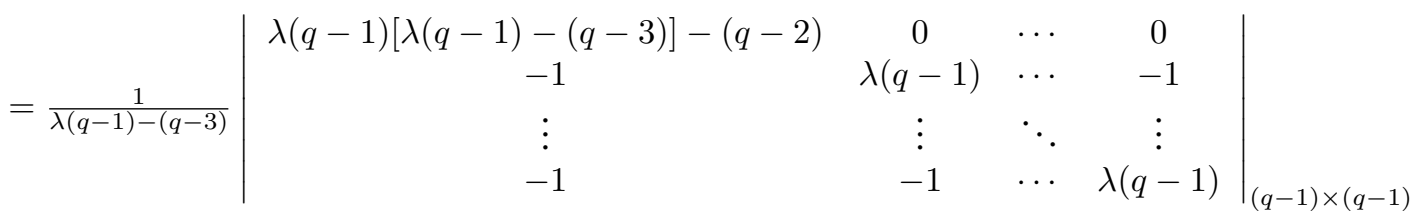

$$
\begin{aligned}
& =\frac{\lambda(q-1)[\lambda(q-1)-(q-3)]-(q-2)}{\lambda(q-1)-(q-3)}\left|\begin{array}{cccc}
\lambda(q-1) & -1 & \cdots & -1 \\
-1 & \lambda(q-1) & \cdots & -1 \\
\vdots & \vdots & \ddots & \vdots \\
-1 & -1 & \cdots & \lambda(q-1)
\end{array}\right|_{(q-2) \times(q-2)} \\
& =\frac{[\lambda(q-1)+1][\lambda(q-1)-(q-2)]}{\lambda(q-1)-(q-3)}\left|\begin{array}{cccc}
\lambda(q-1) & -1 & \cdots & -1 \\
-1 & \lambda(q-1) & \cdots & -1 \\
\vdots & \vdots & \ddots & \vdots \\
-1 & -1 & \cdots & \lambda(q-1)
\end{array}\right|_{(q-2) \times(q-2)} \\
& =\frac{[\lambda(q-1)+1][\lambda(q-1)-(q-2)]}{\lambda(q-1)-(q-3)} \cdot \frac{[\lambda(q-1)+1][\lambda(q-1)-(q-3)]}{\lambda(q-1)-(q-4)}\left|\begin{array}{cccc}
\lambda(q-1) & -1 & \cdots & -1 \\
-1 & \lambda(q-1) & \cdots & -1 \\
\vdots & \vdots & \ddots & \vdots \\
-1 & -1 & \cdots & \lambda(q-1)
\end{array}\right|_{(q-3) \times(q-3)} \\
& =\frac{[\lambda(q-1)+1]^{2}[\lambda(q-1)-(q-2)]}{\lambda(q-1)-(q-4)}\left|\begin{array}{cccc}
\lambda(q-1) & -1 & \cdots & -1 \\
-1 & \lambda(q-1) & \cdots & -1 \\
\vdots & \vdots & \ddots & \vdots \\
-1 & -1 & \cdots & \lambda(q-1)
\end{array}\right|_{(q-3) \times(q-3)}
\end{aligned}
$$

Proceeding like this, we have $A_{1}=[\lambda(q-1)+1]^{q-2}[\lambda(q-1)-(q-2)]$ and hence

$$
\begin{aligned}
\operatorname{det}\left(\lambda I_{q^{n}}-P\right) & =\frac{\left(q^{n}-1\right)^{-1}(q-1)^{-\left(q^{n}-1\right)}}{\lambda(q-1)-(q-2)}\left[\lambda\left(q^{n}-1\right)[\lambda(q-1)-(q-2)]-\left(q^{n}-1\right)\right]\left\{[\lambda(q-1)+1]^{q-2}[\lambda(q-1)-(q-2)]\right\}^{k} \\
& =\left(q^{n}-1\right)^{-1}(q-1)^{-\left(q^{n}-1\right)}\left[\lambda\left(q^{n}-1\right)[\lambda(q-1)-(q-2)]-\left(q^{n}-1\right)\right][\lambda(q-1)+1]^{k(q-2)}[\lambda(q-1)-(q-2)]^{k-1} .
\end{aligned}
$$

Eigenvalues of $P$ are given by the equations,

$$
\begin{aligned}
\lambda^{2}\left(q^{n}-1\right)(q-1)-\lambda\left(q^{n}-1\right)(q-2)-\left(q^{n}-1\right) & =0, \\
\lambda(q-1)+1 & =0, \\
\lambda(q-1)-(q-2) & =0 .
\end{aligned}
$$

Thus, the $q^{n}$ eigenvalues of $P$ are 1 with multiplicity $1,-(q-1)^{-1}$ with multiplicity $k(q-2)+1$ and $(q-2)(q-1)^{-1}$ with multiplicity $k-1$.

Corollary 2.1. Let $F$ be a finite field of order $q$ and $W$ be an n-dimensional vector space over $F$. Then the normalized Laplacian spectrum of the linear dependence graph $\Gamma(W)$ is

$$
\left(\begin{array}{ccc}
0 & q(q-1)^{-1} & (q-1)^{-1} \\
1 & k(q-2)+1 & k-1
\end{array}\right) .
$$

Proof. If the spectrum of $P$ is $\left\{\mu_{1}, \mu_{2}, \ldots, \mu_{n}\right\}$, then the spectrum of $\mathcal{L}(\Gamma(W))$ is $\left\{\left(1-\mu_{1}\right),\left(1-\mu_{2}\right), \ldots,\left(1-\mu_{n}\right)\right\}$. Thus the $q^{n}$ normalized Laplacian eigenvalues of $\Gamma(W)$ are 0 with multiplicity $1,1+(q-1)^{-1}=q(q-1)^{-1}$ with multiplicity $k(q-2)+1$ and $1-(q-2)(q-1)^{-1}=(q-1)^{-1}$ with multiplicity $k-1$. 


\section{The normalized distance Laplacian spectrum of the linear dependence graph $\Gamma(W)$}

In this section, we find the normalized distance Laplacian spectrum of $\Gamma(W)$ in terms of $q$ by using the spectrum of $\bar{P}$.

Theorem 3.1. Let $F$ be a finite field of order $q \geq 2$ and $W$ be an n-dimensional vector space over $F$. Then, the spectrum of $\bar{P}$ is

$$
\left(\begin{array}{ccc}
1 & -\left(2 q^{n}-q-1\right)^{-1} & -q\left(2 q^{n}-q-1\right)^{-1} \\
1 & k(q-2)+1 & k-1
\end{array}\right),
$$

where $k=q^{n-1}+q^{n-2}+\ldots+q+1$.

Proof. In [6], the distance matrix of $\Gamma(W)$ was given as

$$
\mathcal{D}=\left[\begin{array}{cccccccccccccc}
0 & 1 & 1 & \cdots & 1 & 1 & 1 & \cdots & 1 & \cdots & 1 & 1 & \cdots & 1 \\
1 & 0 & 1 & \cdots & 1 & 2 & 2 & \cdots & 2 & \cdots & 2 & 2 & \cdots & 2 \\
1 & 1 & 0 & \cdots & 1 & 2 & 2 & \cdots & 2 & \cdots & 2 & 2 & \cdots & 2 \\
\vdots & \vdots & \vdots & \ddots & \vdots & \vdots & \vdots & \ddots & \vdots & \ddots & \vdots & \vdots & \ddots & \vdots \\
1 & 1 & 1 & \cdots & 0 & 2 & 2 & \cdots & 2 & \cdots & 2 & 2 & \cdots & 2 \\
1 & 2 & 2 & \cdots & 2 & 0 & 1 & \cdots & 1 & \cdots & 2 & 2 & \cdots & 2 \\
1 & 2 & 2 & \cdots & 2 & 1 & 0 & \cdots & 1 & \cdots & 2 & 2 & \cdots & 2 \\
\vdots & \vdots & \vdots & \ddots & \vdots & \vdots & \vdots & \ddots & \vdots & \ddots & \vdots & \vdots & \ddots & \vdots \\
1 & 2 & 2 & \cdots & 2 & 1 & 1 & \cdots & 0 & \cdots & 2 & 2 & \cdots & 2 \\
\vdots & \vdots & \vdots & \vdots & \vdots & \vdots & \vdots & \vdots & \vdots & \ddots & \vdots & \vdots & \vdots & \vdots \\
1 & 2 & 2 & \cdots & 2 & 2 & 2 & \cdots & 2 & \cdots & 0 & 1 & \cdots & 1 \\
1 & 2 & 2 & \cdots & 2 & 2 & 2 & \cdots & 2 & \cdots & 1 & 0 & \cdots & 1 \\
\vdots & \vdots & \vdots & \ddots & \vdots & \vdots & \vdots & \ddots & \vdots & \ddots & \vdots & \vdots & \ddots & \vdots \\
1 & 2 & 2 & \cdots & 2 & 2 & 2 & \cdots & 2 & \cdots & 1 & 1 & \cdots & 0
\end{array}\right]=\left[\begin{array}{ll}
0 \\
\\
\left(J_{\left(q^{n}-1\right) \times 1} \quad 2 J_{\left(q^{n}-1\right)}-\left[J_{q-1}+I_{q-1}\right] \otimes I_{k}\right.
\end{array}\right]
$$

where $k=q^{n-1}+q^{n-2}+\ldots+q+1$. The transmission matrix of $\Gamma(W)$ is given by

$$
T=\left[\begin{array}{cc}
q^{n}-1 & 0_{1 \times\left(q^{n}-1\right)} \\
0_{\left(q^{n}-1\right) \times 1} & \left(2 q^{n}-q-1\right) I_{q-1} \otimes I_{k}
\end{array}\right]
$$

where $k=q^{n-1}+q^{n-2}+\ldots+q+1$. Hence,

$$
\begin{aligned}
\bar{P}=T^{-1} \mathcal{D} & =\left[\begin{array}{cc}
\left(q^{n}-1\right)^{-1} & 0_{1 \times\left(q^{n}-1\right)} \\
0_{\left(q^{n}-1\right) \times 1} & \left(2 q^{n}-q-1\right)^{-1} I_{q-1} \otimes I_{k}
\end{array}\right]\left[\begin{array}{cc}
0 & J_{1 \times\left(q^{n}-1\right)} \\
J_{\left(q^{n}-1\right) \times 1} & 2 J_{\left(q^{n}-1\right)}-\left(\left[J_{q-1}+I_{q-1}\right] \otimes I_{k}\right)
\end{array}\right] \\
& =\left[\begin{array}{cc}
0 & \left(q^{n}-1\right)^{-1} J_{1 \times\left(q^{n}-1\right)} \\
\left(2 q^{n}-q-1\right)^{-1} J_{\left(q^{n}-1\right) \times 1} & \left(2 q^{n}-q-1\right)^{-1}\left[2 J_{\left(q^{n}-1\right)}-\left(\left[J_{q-1}+I_{q-1}\right] \otimes I_{k}\right)\right]
\end{array}\right] .
\end{aligned}
$$

The characteristic polynomial of $\bar{P}$ is

$$
\begin{aligned}
\operatorname{det}\left(\lambda I_{q^{n}}-\bar{P}\right) & =\left|\begin{array}{cc}
\lambda & -\left(q^{n}-1\right)^{-1} J_{1 \times\left(q^{n}-1\right)} \\
-\left(2 q^{n}-q-1\right)^{-1} J_{\left(q^{n}-1\right) \times 1} & \lambda I_{q^{n}-1}-2\left(2 q^{n}-q-1\right)^{-1} J_{q^{n}-1}+\left[\left(2 q^{n}-q-1\right)^{-1}\left(J_{q-1}+I_{q-1}\right) \otimes I_{k}\right]
\end{array}\right| \\
& =\left(\begin{array}{ll}
\left.q^{n}-1\right)^{-1}\left[\left(2 q^{n}-q-1\right)^{-1}\right]^{\left(q^{n}-1\right)} & -J_{1 \times\left(q^{n}-1\right)} \\
-J_{\left(q^{n}-1\right) \times 1} & \lambda\left(2 q^{n}-q-1\right) I_{q^{n}-1}-2 J_{q^{n}-1}+\left[\left(J_{q-1}+I_{q-1}\right) \otimes I_{k}\right]
\end{array} \mid .\right.
\end{aligned}
$$

Now, apply the elementary transformations of subtracting from each column $C_{i}$, twice of $C_{1}$ for $i=2,3, \ldots, q^{n}$, result in

$$
\begin{aligned}
\operatorname{det}\left(\lambda I_{q^{n}}-\bar{P}\right) & =\left(q^{n}-1\right)^{-1}\left[\left(2 q^{n}-q-1\right)^{-1}\right]^{\left(q^{n}-1\right)}\left|\begin{array}{cc}
\lambda\left(q^{n}-1\right) & -\left[1+2 \lambda\left(q^{n}-1\right)\right] J_{1 \times\left(q^{n}-1\right)} \\
-J_{\left(q^{n}-1\right) \times 1} & \left(\left[\lambda\left(2 q^{n}-q-1\right)+1\right] I_{q-1}+J_{q-1}\right) \otimes I_{k}
\end{array}\right| \\
& =\left(q^{n}-1\right)^{-1}\left[\left(2 q^{n}-q-1\right)^{-1}\right]^{\left(q^{n}-1\right)}\left[1+2 \lambda\left(q^{n}-1\right)\right]\left|\begin{array}{cc}
\frac{\lambda\left(q^{n}-1\right)}{\left(1+2 \lambda\left(q^{n}-1\right)\right)} & -J_{1 \times\left(q^{n}-1\right)} \\
-J_{\left(q^{n}-1\right) \times 1} & \left(\left[\lambda\left(2 q^{n}-q-1\right)+1\right] I_{q-1}+J_{q-1}\right) \otimes I_{k}
\end{array}\right| .
\end{aligned}
$$

Multiplying the first row by $\lambda\left(2 q^{n}-q-1\right)+q$ and $R_{1}^{\prime} \mapsto R_{1}^{\prime}+R_{2}+\ldots+R_{q^{n}}$ yield

$\operatorname{det}\left(\lambda I_{q^{n}}-\bar{P}\right)=$

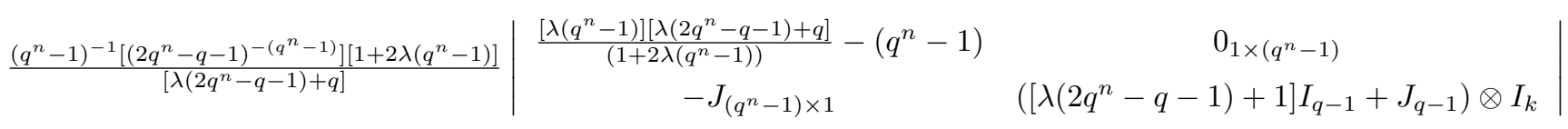


$=\frac{\lambda\left(q^{n}-1\right)\left[\lambda\left(2 q^{n}-q-1\right)+q\right]-\left(q^{n}-1\right)\left(1+2 \lambda\left(q^{n}-1\right)\right)}{\left(q^{n}-1\right)\left(2 q^{n}-q-1\right)^{\left(q^{n}-1\right)}\left[\lambda\left(2 q^{n}-q-1\right)+q\right]} \operatorname{det}\left(\left[\lambda\left(2 q^{n}-q-1\right)+1\right] I_{q-1}+J_{q-1}\right)^{k}$ using Lemma1.1.

If $B_{1}=\operatorname{det}\left[\lambda\left(2 q^{n}-q-1\right)+1\right] I_{q-1}+J_{q-1}$ then

$B_{1}=\left|\begin{array}{cccc}\lambda\left(2 q^{n}-q-1\right)+2 & 1 & \cdots & 1 \\ 1 & \lambda\left(2 q^{n}-q-1\right)+2 & \cdots & 1 \\ \vdots & \vdots & \ddots & \vdots \\ 1 & 1 & \cdots & \lambda\left(2 q^{n}-q-1\right)+2\end{array}\right|_{(q-1) \times(q-1)}$

Multiply first row by $\lambda\left(2 q^{n}-q-1\right)+(q-1)$ and then $R_{1}^{\prime} \mapsto R_{1}^{\prime}-R_{2}-\ldots-R_{q-1}$, we have

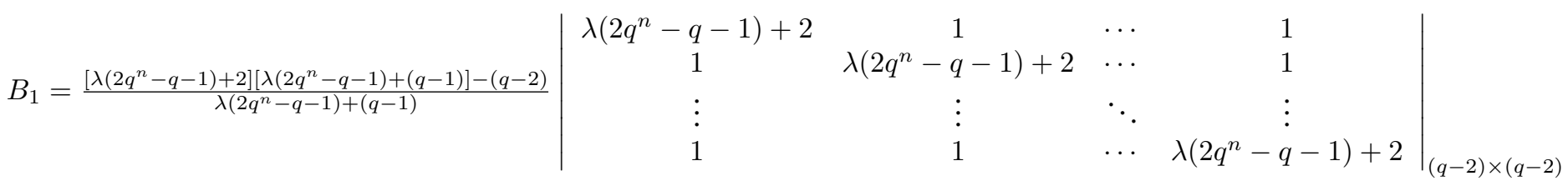

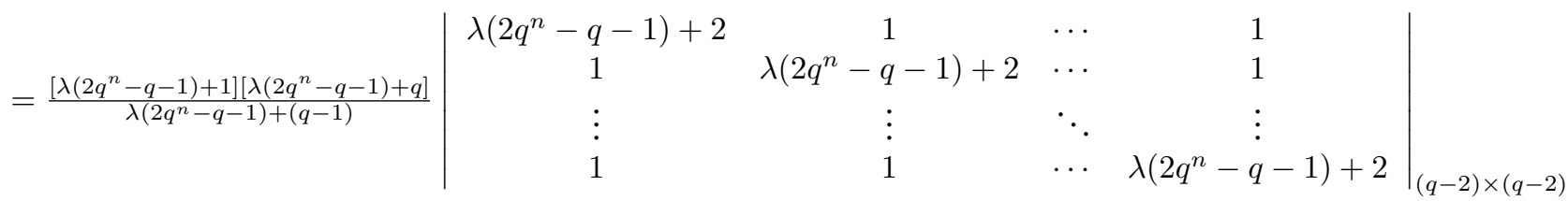

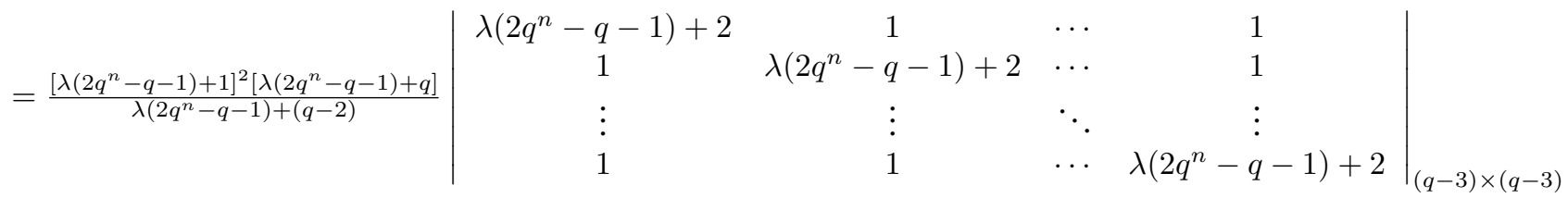

Proceeding like this, we have $B_{1}=\left[\lambda\left(2 q^{n}-q-1\right)+1\right]^{(q-2)}\left[\lambda\left(2 q^{n}-q-1\right)+q\right]$ and hence,

$$
\operatorname{det}\left(\lambda I_{q^{n}}-\bar{P}\right)=\frac{\lambda\left(q^{n}-1\right)\left[\lambda\left(2 q^{n}-q-1\right)+q\right]-\left(q^{n}-1\right)\left(1+2 \lambda\left(q^{n}-1\right)\right)}{\left(q^{n}-1\right)\left(2 q^{n}-q-1\right)^{\left(q^{n}-1\right)}\left[\lambda\left(2 q^{n}-q-1\right)+q\right]}\left[\lambda\left(2 q^{n}-q-1\right)+1\right]^{k(q-2)}\left[\lambda\left(2 q^{n}-q-1\right)+q\right]^{k}
$$

The right hand side of the above equation is equal to

$$
\frac{\left[\lambda^{2}\left(q^{n}-1\right)\left(2 q^{n}-q-1\right)+\lambda\left(q^{n}-1\right)\left(q-2\left(q^{n}-1\right)\right)-\left(q^{n}-1\right)\right]}{\left(q^{n}-1\right)\left(2 q^{n}-q-1\right)^{\left(q^{n}-1\right)}}\left[\lambda\left(2 q^{n}-q-1\right)+1\right]^{k(q-2)}\left[\lambda\left(2 q^{n}-q-1\right)+q\right]^{k-1} .
$$

Thus, the eigenvalues of $\bar{P}$ are given by the following equations

$$
\begin{aligned}
\lambda^{2}\left(q^{n}-1\right)\left(2 q^{n}-q-1\right)+\lambda\left(q^{n}-1\right)\left(q-2\left(q^{n}-1\right)\right)-\left(q^{n}-1\right) & =0, \\
{\left[\lambda\left(2 q^{n}-q-1\right)+1\right]^{k(q-2)} } & =0, \\
{\left[\lambda\left(2 q^{n}-q-1\right)+q\right]^{k-1} } & =0 .
\end{aligned}
$$

After solving the above equations, we conclude that the $q^{n}$ eigenvalues of $\bar{P}$ are 1 with multiplicity $1,-\left(2 q^{n}-q-1\right)^{-1}$ with multiplicity $k(q-2)+1$ and $-q\left(2 q^{n}-q-1\right)^{-1}$ with multiplicity $k-1$ respectively.

Corollary 3.1. Let $F$ be a finite field of order $q \geq 2$ and $W$ be an n-dimensional vector space over $F$. Then the normalized distance Laplacian spectrum of the linear dependence graph $\Gamma(W)$ is

$$
\left(\begin{array}{ccc}
0 & 1+\left(2 q^{n}-q-1\right)^{-1} & 1+q\left(2 q^{n}-q-1\right)^{-1} \\
1 & k(q-2)+1 & k-1
\end{array}\right) .
$$

Proof. If the spectrum of $\bar{P}$ is $\left\{\mu_{1}^{\prime}, \mu_{2}^{\prime}, \ldots, \mu_{n}^{\prime}\right\}$, then the spectrum of $\mathcal{D}^{N}(\Gamma(W))$ is $\left\{\left(1-\mu_{1}^{\prime}\right),\left(1-\mu_{2}^{\prime}\right), \ldots,\left(1-\mu_{n}^{\prime}\right)\right\}$. Thus, the $q^{n}$ normalized distance Laplacian eigenvalues of $\Gamma(W)$ are 0 with multiplicity $1,1+\left(2 q^{n}-q-1\right)^{-1}$ with multiplicity $k(q-2)+1$ and $1+q\left(2 q^{n}-q-1\right)^{-1}$ with multiplicity $k-1$ respectively.

\section{Conclusion}

In this paper, we obtained the normalized Laplacian and normalized distance Laplacian spectra of the linear dependence graph $\Gamma(W)$ of a finite-dimensional vector space $W$ over a finite field $F$. These spectra are the new addition to the existing literature of spectral graph theory. A similar treatment can be applied to obtain other spectra associated with other matrices of a linear dependence graph. 


\section{References}

[1] M. Aouchiche, P. Hansen, Two Laplacians for the distance matrix of a graph, Linear Algebra Appl. 439 (2013) 21-33.

[2] D. M. Cvetković, M. Doob, H. Sachs, Spectra of Graphs - Theory and Applications, Academic Press, 1980.

[3] F. R. K. Chung, F. C. Graham, Spectral Graph Theory, American Mathematical Society, 1997.

[4] F. Ali, Y. Li, The connectivity and the spectral radius of commuting graphs on certain finite groups, Linear Multilinear Algebra, DOI: 10.1080/03081087.2019.1700893, In press.

[5] E. Hückel, Quantentheoretische beitrage zum benzolproblem, Z. Phys. 70 (1931) 204-286.

[6] S. Maity, A. K. Bhuniya, On the spectrum of linear dependence graph of a finite dimensional vector space, Electron. J. Graph Theory Appl. 7 (2019) $43-59$.

[7] C. Reinhart, The normalized distance Laplacian, arXiv:1903.04575v2 [math.CO] (2019).

[8] M. Torktaz, A. R. Ashrafi, Spectral properties of the commuting graphs of certain groups, AKCE Int. J. Graphs Comb. 16 (2019) 300-309.

[9] Z. Mehranian, A. Gholami, A. R. Ashrafi, The Spectra of power graphs of certain finite groups, Linear Multilinear Algebra 65 (2017) 1003-1010.

[10] U. Hayat, A. N. Celis, F. Ali, Commuting graphs on Coxeter groups, Dynkin diagrams and finite subgroups of $S L(2, \mathbb{C})$, arXiv:1703.02480v2 [math.GR] (2017)

[11] S. Abdussakir, M. N. Jauhari, F. Ali, Survey on topological indices and graphs associated with a commutative ring, J. Phys.: Conference Series 1562 (2020) Art\# 012008. 